\title{
Study of Electrical Properties of Ferrofluids
}

\author{
M.UNIYAL ${ }^{1}$, SURESH C. NAUTIYAL ${ }^{2 *}$, SHAURYA BHATT ${ }^{1}$ and S. C. BHATT ${ }^{1}$ \\ ${ }^{1}$ Department of Physics, \\ H N B Garhwal University, Srinagar Garhwal, Uttarakhand- 246174, India \\ ${ }^{2}$ Govt. Degree College Raipur, Dehradun-248001, India \\ scbhattin@yahoo.com
}

Received 19 May 2018 / Accepted 10 June 2018

\begin{abstract}
Study of electrical properties of ferrofluid have been carried out on a water based ferrofluid, dielectric constant, loss tangent and electrical conductivity have been measured with the help of a R C L meter at $0.1,1,10,100$ and $1000 \mathrm{KHz}$ frequencies at room temperature. The results have been plotted in the graphs and discussed.
\end{abstract}

Keywords: Ferrofluids, Dielectric constant, Loss tangent, Cole-Cole plot

\section{Introduction}

A ferrofluid is a fluid comprising colloidal suspension of ultra fine magnetic particles of the order of the range $10-1000^{\circ} \mathrm{A}$ size. To prevent each magnetic grain from clustering together and to maintain a true colloidal dispersion, it is coated with suitable surfactants like oleic acid and some fatty acids. Ferrofluids are widely used in many engineering devices such as magnetic seal, dampers and sensors ${ }^{1,2}$. Ferrofluids are also found to be useful for many biomedical applications ${ }^{3,4}$.

A ferrofluid is a stable colloidal dispersion of magnetic particles. This is due to delicate balance of attractive and repulsive forces between particles. In particular the interaction between magnetic moments in neighboring particles gives rise to magnetic forces and the surfactant molecules attached to their surface.

Ferro fluids show a change in their magnetic, thermo physical, mechanical, optical and acoustic properties in the presence of a magnetic field ${ }^{5}$.

Ferrofluids contain single domain magnetic particles whose magnetic moments may align with an applied magnetic field by two distinct mechanisms- either bulk rotation of the particles with its magnetic moments locked in an easy direction of magnetization or rotation of the magnetic vector out of the easy direction. The later process is known as Neel rotation and has a relaxation time given ${ }^{6}$.

$$
\tau_{\mathrm{n}}^{-1}=\mathrm{f}_{\mathrm{o}} \exp (-\mathrm{K} V / \mathrm{K} \mathrm{T})
$$


Where $\mathrm{K}$ is the effective anisotropy constant of the particle and V is its volume, $\mathrm{f}_{\mathrm{o}}$ has dimension of frequency ${ }^{7}$. The bulk rotation mechanism has a relaxation time given by ${ }^{8}$

$$
\tau_{\mathrm{B}}=3 \mathrm{~V} \eta / \mathrm{K} \mathrm{T}
$$

Where $\eta$ is the viscosity of the carrier liquid.

The dominant magnetization process of a particle will be that which has the shortest relaxation time. Ferrofluids invariably contain a distribution of particles sizes and in general both mechanisms will contribute to the value of magnetization.

If a magnetic fluid is frozen in zero applied fields, then relaxation of the magnetic moments of the particles can only occur by Neel rotation. The Neel relaxation time is rapidly varying function of the particle diameter $\mathrm{D}\left(\mathrm{V}=\mathrm{D}^{3} / 6\right)$ and this leads to the concept of blocked particles ${ }^{9}$ i.e., above a critical diameter Dp, the moment cannot relax in the time taken for a measurement of magnetization to be made and below Dp the moment relaxes within the time of measurement. Particles with $\mathrm{D}<=\mathrm{D}_{\mathrm{p}}$ are called super paramagnetic and only these particles contribute significantly to the magnetization of the sample in small applied fields ${ }^{10}$.

The universal properties of ferrofluids propose some unique applications, in particular in connection with seals, pumps, printing and surgery ${ }^{11}$. There exist a distribution of particle sizes in a ferrofluid and determination of this distribution would clarify understanding and prediction of the fluids properties. The suspended single domain Ferro fluid particles have diameters typically $\leq 150^{\circ} \mathrm{A}$ and are therefore super paramagnetic at room temperature.

Scaife $^{12}$ suggested the polarizability is a good measure of intrinsic properties of a substance, i.e., when the dielectric constant becomes complex quantity, the dielectric constant and dielectric loss are given by the Debye equations -

$$
\begin{aligned}
\varepsilon^{\prime} & =\varepsilon_{\infty}+\frac{\varepsilon_{0}-\varepsilon_{\infty}}{1+\omega^{2} \lambda^{2}} \\
\varepsilon^{\prime \prime} & =\frac{\varepsilon_{0}-\varepsilon_{\infty}}{1+\omega^{2} \lambda^{2}}
\end{aligned}
$$

The polarizability also becomes a complex polarizability $\alpha *(\omega)$ and may be written as -

$$
\alpha^{*}(\omega)=\alpha^{\prime}-i \alpha^{\prime \prime}=\frac{\varepsilon^{*}-1}{\varepsilon^{*}-2}
$$

Cole-Cole ${ }^{13}$ suggested that if $\epsilon$ ' and $\epsilon$ " are plotted the plot is a semi circle. Using above equations real and imaginary parts of polarizability can be found, which also follow a Cole-Cole relation.

\section{Experimental}

A water based ferrofluid FW40 from Marpomagna Co Japan is used. The ferrofluid consists of $\mathrm{Fe}_{3} \mathrm{O}_{4}$ particles of mean diameter $8 \mathrm{~nm}$ in a water medium and has a saturation magnetization of 400 Gauss and density $1.359 \mathrm{~g} / \mathrm{m} 3$. The RCL meter PM6306 is used to measure the rheological properties. The PM 6306 RCL meter is used for precise measurements of resistance, capacitance and inductance. Its basic accuracy is $0.1 \%$. It allows fast and high precision measurements and diagnostic of passive components over a wide range. A micro-processor controls the measurement process, computes the measurement value and transfer the result to the display. 


\section{Results and Discussion}

The frequency dependence of dielectric constant, loss tangent and electrical conductivity has been shown in Figure 1-3 respectively. From the Figs it observed that the dielectric constant is higher at lower frequency as the frequency is increased, dielectric constant decreases, showing the presence of electronic and ionic polarization. The loss tangent, however, increases with the increase of frequency and after $1 \mathrm{MHz}$ it decreases with the increase of frequency as is evident from Figure 2. Electrical conductivity decreases with the increase of frequency, as is shown in Figure 3. Dielectric constant depends on electronic, ionic, dipole and space charge polarization. Contributions due to electronic and ionic polarization are frequency dependent, whereas dipole and space charge contributions are frequency dependent at low frequencies. Figure 4, shows a Cole-Cole plot of polarizability for a water base ferrofluid, which shows an approximate semi circle.

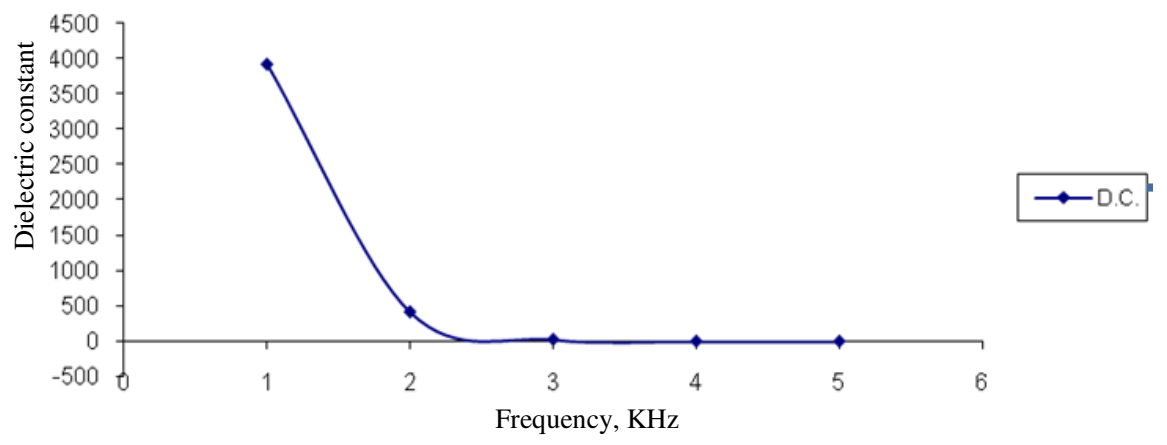

Figure 1. Frequency dependence of dielectric constant

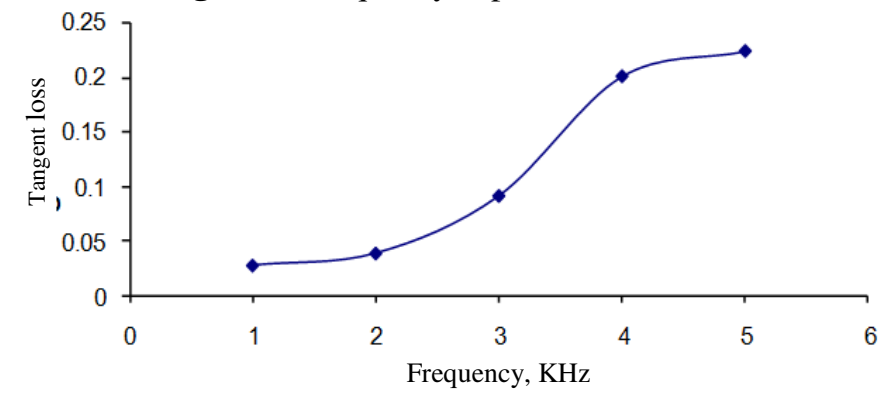

Figure 2. Frequency dependence of tangent loss

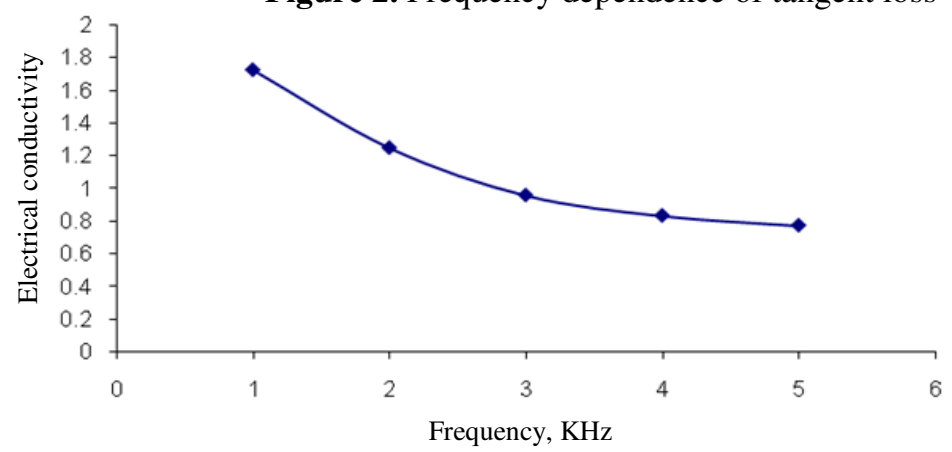

Figure 3. Frequency dependence of electrical conductivity 


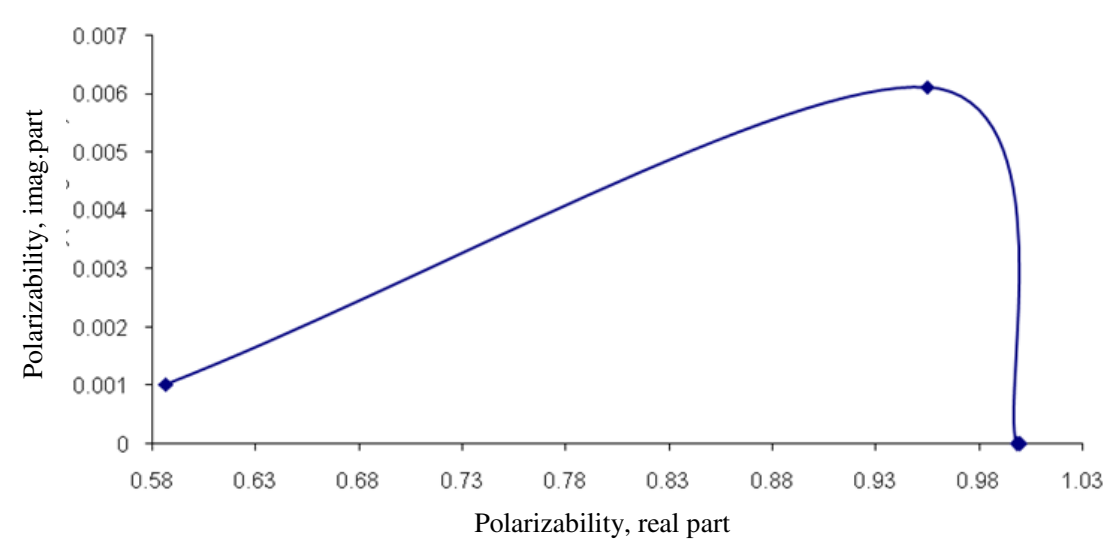

Figure 4. Cole-Cole plot of polarizability for a water based ferrofluid

\section{References}

1. Mehta R V, J Sci Ind Res., 1985, 44, 580.

2. Raj K and Inoskwitz, J Magn Magn Mater, 1990, 85(1-3), 233; DOI:10.1016/03048853(90)90058-X

3. Rusetski A N and Ruuge E K, J Magn Magn Mater., 1990, 85(1-3), 299-302; DOI:10.1016/0304-8853(90)90070-7

4. Charles S W, J Magn Magn Mater., 1990, 85(1-3), 277-284; DOI:10.1016/03048853(90)90066-Y

5. Chikazumi S, Taketomi S, Ukita M, Mizukami M, Miyajima H, Ssetsgawa M and Kurihara Y, J Magn Magn Mater., 1987, 65(2-3), 245-251; DOI:10.1016/03048853(87)90043-6

6. Neel L, Comp Rend Acad Sci., 1949, 228, 664.

7. Kneller E, Proc.of the Intern.Conf.on magnetism, Nottingham(1964)p.174.

8. Shliomis M T, Sov Phys USP, 1974, 17, 153-169.

9. Bean C P and Livingston J D, J Appl Phys., 1959, 30(12), 1205; https://doi.org/10.1063/1.1735100

10. Wohlforth E P, Phys Lett A, 1979, 70(5-6), 489-491; DOI:10.1016/03759601(79)90375-X

11. J Potton A, Daniell G J, Eastop A D, Kitching M, Melville D, Poslad S, Rainford B D and Stanley H, J Magn Magn Mater., 1983, 39(1-2), 95-98; DOI:10.1016/03048853(83)90408-0

12. Scaife B K, Proc Phys Soc London(G.B.), 1963, 81, 124.

13. Cole K S and Cole R H, J Chem Phys., 1941, 9(4), 341; DOI:10.1063/1.1750906 